\title{
Universality of the Empty-Lattice Approximation to Predict the Topology of Energy Spectra of High-Symmetry Crystals and Superlattices Based upon Them
}

\author{
M. SznAjder ${ }^{a, *}$, D.M. BerchA ${ }^{a}$, K.E. Glukhov ${ }^{b}$ \\ AND I.V. SLIPUKHINA ${ }^{b}$ \\ ${ }^{a}$ Institute of Physics, University of Rzeszów \\ Rejtana 16a, 35-310 Rzeszów, Poland \\ ${ }^{b}$ Institute of Physics and Chemistry of Solid State \\ Uzhgorod National University \\ 54 Voloshin St., 88000 Uzhgorod, Ukraine

\begin{abstract}
$O_{h}^{7}, T_{d}^{2}$ and $O_{h}^{5}$ symmetry crystals were discussed to demonstrate universality of the empty-lattice approximation to obtain the topology and symmetry of the elementary energy bands creating the valence band of those crystals and to predict a localization of the maximum of valence electron density distribution in the unit cell. The elaborated concept of the elementary energy bands was applied to the $(\mathrm{GaAs})_{5} /(\mathrm{AlAs})_{5}$ superlattice and ordered solid solution $\mathrm{Pb}_{0.5} \mathrm{Sn}_{0.5} \mathrm{~S}$.
\end{abstract}

PACS numbers: 71.20.Nr, 71.15.Mb, 71.20.Mq, 73.21.Cd, 61.50.Ah

\section{Introduction}

It has been demonstrated in the literature that the band structure of crystals is composed of some simplest elements, i.e. elementary energy bands [1-4]. The elementary energy bands were obtained by Zak for a given space symmetry group describing a crystal by inducing space group representations for high-symmetry points in the Brillouin zone (BZ) from irreducible representations of the site-symmetry group of a certain Wyckoff position which is assigned to the considered space group. Zak utilized at the same time the idea of the continu-

*corresponding author; e-mail: sznajder@univ.rzeszow.pl 
ity chords of bands in the $k$ space [1]. Since there are more than one Wyckoff position assigned to the most space symmetry groups and the dimension of the site-symmetry groups of those positions can be 1,2, 3 or 4 , therefore, the elementary energy bands induced for a given space group can have various topologies. Moreover, there is an ambiguity in the choice of the Wyckoff position for the induction procedure.

We showed that there exists a simple method to distinguish the actual Wyckoff position, i.e. such one for which the elementary energy bands induced from a certain site-symmetry group representation create the valence band of semiconducting crystals $[5,6]$. This method is the simplest solid state approximation, namely, the empty-lattice approximation, supplied with general data concerning a semiconductor (the existence of the forbidden energy gap, lattice constants, number of valence electrons in the unit cell, space symmetry group). The distinguished actual Wyckoff position has a physical meaning, there is a maximum of the spatial valence electron density distribution focused in this position in the unit cell. The method of selection of the actual Wyckoff position has been checked for the orthorhombic crystals, since in this case, the site-symmetry group representations of the Wyckoff positions are one-dimensional and, as a consequence, a topology of the elementary energy bands is the same for crystals belonging to the same orthorhombic space symmetry group.

We intend to investigate in this paper whether the empty-lattice approximation is a sufficient method to find the actual Wyckoff position for high-symmetry crystals $\left(O_{h}^{7}, T_{d}^{2}, O_{h}^{5}\right)$, for which the site-symmetry group representations are multi-dimensional, and to predict at the same time the topology and symmetry of the valence band of those crystals. The multi-dimensional representations lead to the elementary energy bands with various numbers of branches within the same space group. For this purpose, in the first part of the article, we discuss cubic crystals and then, in the second part, we utilize our method to distinguish, based upon the empty-lattice approximation, the actual Wyckoff position that is responsible for the spatial valence electron distribution and the creation of the valence band of superlattices and ordered solid solutions.

\section{Elementary energy bands for crystals having $O_{h}^{7}, T_{d}^{2}, O_{h}^{5}$ symmetry}

A classical crystal described by the space group $O_{h}^{7}$ is silicon. Since this is the most investigated semiconductor, it is the best one to verify our method, using literature data. The sequence of energy states obtained in the empty-lattice approximation is as follows [7]:

$$
\begin{array}{ll}
\text { point } \Gamma & \Gamma_{1},\left(\Gamma_{25}^{\prime} \downarrow \Gamma_{1} \Gamma_{15} \Gamma_{2}^{\prime}\right),\left(\Gamma_{25}^{\prime} \Gamma_{12}^{\prime} \Gamma_{2}^{\prime}\right), \ldots, \\
\text { point } X\left(\frac{2 \pi}{a}, 0,0\right) & X_{1},\left(X_{4} \downarrow X_{1}\right),\left(X_{1} X_{2} X_{3} X_{4}\right), \ldots, \\
\text { point } L\left(\frac{\pi}{a}, \frac{\pi}{a}, \frac{\pi}{a}\right) & \left(L_{1} L_{2}^{\prime}\right),\left(L_{3}^{\prime} \downarrow L_{1} L_{2}^{\prime} L_{3}\right), \ldots
\end{array}
$$


It appears that the real valence band of silicon is composed of the states given by Eq. (1) at the left side of the arrows showing the energy position of the forbidden gap [7]. These states, selected by the empty-lattice approximation, exist in the elementary energy band which create the valence band of silicon: $X_{1} \oplus X_{4}-\Gamma_{1} \oplus$ $\Gamma_{25}^{\prime}-L_{1} \oplus L_{2}^{\prime} \oplus L_{3}^{\prime}$. Considering all eight Wyckoff positions assigned to the $O_{h}^{7}$ space group [8] we come to a conclusion that the set of representations creating this elementary energy band corresponds to the unity representation $\tau_{1}$ of the sitesymmetry group of the Wyckoff position $c(1 / 8,1 / 8,1 / 8)$. According to our idea there is a maximum of the valence electron density distribution in the Wyckoff position selected by the empty-lattice approximation. A fact that a maximum of the valence electron density distribution exists in the middle between neighbouring silicon atoms has been well known for a long time. Hence, the Wyckoff position $c(1 / 8,1 / 8,1 / 8)$ is the actual one for the $O_{h}^{7}$ space group.

An important representative of $T_{d}^{2}$ symmetry semiconductors is gallium arsenide. Calculation results of the energy states obtained in the empty-lattice approximation for GaAs show a set of the following elementary energy bands:

$$
X_{1}-\Gamma_{1}-L_{1}, \quad X_{3} \oplus X_{5}-\Gamma_{15}-L_{1} \oplus L_{3} .
$$

These two sets describe at the same time the whole valence band of GaAs and other representatives of $\mathrm{A}^{\mathrm{III}} \mathrm{B}^{\mathrm{V}}$ group, furthermore, there is an energetical gap between $X_{1}$ and $X_{3}$ states which is not observed in the valence band of $\mathrm{A}^{\mathrm{IV}}$ crystals. The presence of this gap is a result of the symmetry decrease from $O_{h}^{7}$ up to $T_{d}^{2}$ as a consequence of two different atoms present in the unit cell. We regard these two elementary energy bands (2) as physically related ones. Now, one should distinguish between 8 Wyckoff positions assigned to the $T_{d}^{2}$ group the actual one, which is responsible for the described symmetry and topology of the valence band of GaAs. Conducting the induction procedure of the $T_{d}^{2}$ space group representations from the irreducible representations of the site-symmetry group of all 8 Wyckoff positons [9] we conclude that the set of physically related elementary energy bands (2) is obtained from the unity representation of the site-symmetry group of the Wyckoff position $e(x, x, x)$. This position is a line connecting $\mathrm{Ga}$ and As atoms along the main diagonal in a cubic cell and it has one degree of freedom compared to the actual Wyckoff position of the silicon crystal. Therefore we will call it the itinerant Wyckoff position. According to the idea described above, there is a maximum of the valence electron density focused in this position. The itinerant Wyckoff position points out a displacement of the maximum of the valence electron density from the cation to the anion in comparison with a situation observed for silicon. Such charge asymmetry distribution for GaAs is also described in the literature [10].

One of the representatives of $O_{h}^{5}$ group crystal is the PbS semiconductor, exhibiting the natrium chloride structure. The energy states of this crystal obtained in the empty-lattice approximation, in the order of increasing energies are as following: 


$$
\begin{array}{ll}
\text { point } \Gamma & \Gamma_{1},\left(\Gamma_{1} \Gamma_{15} \downarrow \Gamma_{25}^{\prime} \Gamma_{2}^{\prime}\right), \ldots, \\
\text { point } X\left(0,0, \frac{2 \pi}{a}\right) & \left(X_{1} X_{4}\right),\left(X_{1} X_{10} \downarrow X_{7}\right),\left(X_{1} X_{4} X_{5} X_{8} X_{9} X_{10}\right), \ldots, \\
\text { point } L\left(\frac{\pi}{a}, \frac{\pi}{a}, \frac{\pi}{a}\right) & \left(L_{1} L_{2}\right),\left(L_{1} L_{3} \downarrow L_{2}^{\prime} L_{3}\right),\left(L_{1} L_{3}^{\prime} L_{3} L_{2}^{\prime}\right), \ldots
\end{array}
$$

Arrows show positions where the forbidden energy gap will be created under an influence of the crystalline potential. Hence, the valence band of $\mathrm{PbS}$ is created from the set of states: $\left(2 \Gamma_{1}, \Gamma_{15}\right)-\left(2 X_{1}, X_{4}, X_{10}\right)-\left(2 L_{1}, L_{2}^{\prime}, L_{3}\right)$. Analysing all 11 Wyckoff positions for the $O_{h}^{5}$ space group we come to a conclusion that this set is composed of three elementary energy bands corresponding to the Wyckoff positions $a(0,0,0): \Gamma_{1}-X_{1}-L_{1}$ and $b(1 / 2,1 / 2,1 / 2): \Gamma_{1}-X_{1}-L_{2}^{\prime}, \Gamma_{15}-X_{4} \oplus X_{10}-L_{1} \oplus L_{3}$, respectively. Hence, as opposed to orthorhombic and cubic crystals from $O_{h}^{7}$ and $T_{d}^{2}$ group, the actual Wyckoff positions for $O_{h}^{5}$ group should be regarded as two positions $a(0,0,0)$ and $b(1 / 2,1 / 2,1 / 2)$. This means that not only the valence electrons of the anion $S$ situated in the Wyckoff position $b$ are responsible for the creation of the valence band, but also electrons of the cation $\mathrm{Pb}$ situated in the position $a$.

\section{Influence of the symmetry decrease and enlargement of the unit cell on evolution of the elementary energy bands}

The symmetry of a crystal can be lowered due to a phase transition and this process can proceed with $n$-times enlargement of the unit cell. However, such situation can be obtained in an artificial way, i.e. by creating a superlattice based upon cubic crystals. We will consider below the $(\mathrm{GaAs})_{5} /(\mathrm{AlAs})_{5}$ superlattice. Its lattice constants are $a=3.9258 \AA, c=28.201 \AA$ in an equilibrium state and the symmetry group is $D_{2 d}^{5}$. The unit cell contains 20 atoms and hence 80 valence electrons. Therefore 40 minibands are filled in the valence band of this superlattice. Calculating the energy states of $(\mathrm{GaAs})_{5} /(\mathrm{AlAs})_{5}$ in the emptylattice approximation we conclude that the closed valence band, after a smallest possible rearrangement of states in the $\Gamma$ point is composed of the sets

$$
\begin{aligned}
& 14\left(\Gamma_{1}, \Gamma_{3}\right)+6 \Gamma_{5}-17\left(X_{1}, X_{3}\right)+3\left(X_{2}, X_{4}\right)-3\left(M_{1}, M_{3}\right) \\
& \quad+3\left(M_{2}, M_{4}\right)+14 M_{5} .
\end{aligned}
$$

High-symmetry points of $(\mathrm{GaAs})_{5} /(\mathrm{AlAs})_{5}$ are presented in Fig. 1.

Inducing representations of the $D_{2 d}^{5}$ space group from irreducible representations of the site-symmetry groups of all 11 Wyckoff positions for $D_{2 d}^{5}$ we conclude that Eq. (4) can be rebuilt using the elementary energy bands originating from $g(0,1 / 2, z)$. The site-symmetry group of this position has 4 irreducible representations $\tau_{i}(i=1,2,3,4)$ and the requested elementary energy bands are obtained by the induction procedure from $\tau_{1}, \tau_{3}$, and $\tau_{4}$ as follows:

$$
\begin{aligned}
14 \tau_{1} & +3 \tau_{3}+3 \tau_{4}: \\
& 14\left(\Gamma_{1} \oplus \Gamma_{3}\right)+3 \Gamma_{5}+3 \Gamma_{5}-14\left(X_{1} \oplus X_{3}\right) \\
& +3\left(X_{1} \oplus X_{4}\right)+3\left(X_{2} \oplus X_{3}\right)-14 M_{5}+3\left(M_{1} \oplus M_{3}\right)+3\left(M_{2} \oplus M_{4}\right) .
\end{aligned}
$$




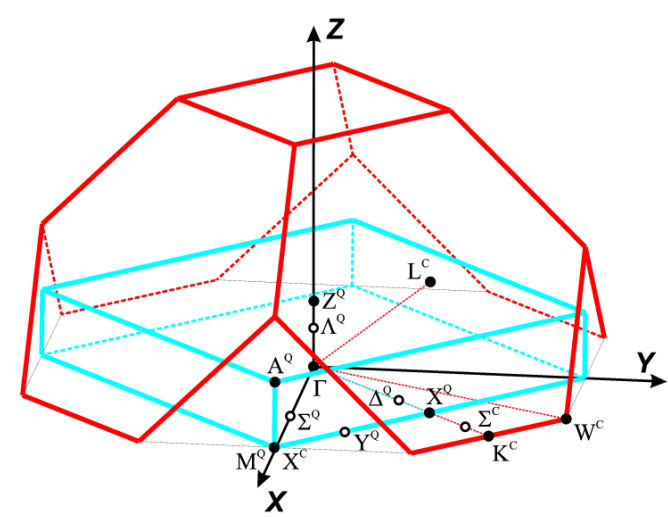

Fig. 1. Correspondence between the high-symmetry points in the BZs of the $(\mathrm{GaAs})_{5} /(\mathrm{AlAs})_{5}$ superlattice (superscript "Q") and of the GaAs crystal (superscript "C").

Hence, the empty-lattice approximation distinguishes the Wyckoff position $g$ (and its multiplicity $g_{1}$ and $g_{2}$ ) which represent lines along the stacking axis of the superlattice. Along these lines, there should be electron density maintaining as a whole the created unit cell of a super-crystal. However, this position $g$ does not give yet information about the initial bondings of materials which create the superlattice. Since the folding procedure of the crystal spectrum into a miniband superlattice spectrum contracts the valence band branches, hence, based upon the empty-lattice approximation, one can predict only a topology of the elementary energy bands creating the valence band, given by Eq. (5), however the coefficients near the corresponding sets of representations can be different for the real numerical band structure calculations.

We conducted $a b$ initio band structure calculations of the $(\mathrm{GaAs})_{5} /(\mathrm{AlAs})_{5}$ superlattice in the framework of the Hohenberg-Kohn-Sham density functional theory $[11,12]$ in the LDA approximation [13] by means of the ABINIT code [14]. The special $\boldsymbol{k}$ points method [15] was used to carry out integration in the $k$ space over the BZ and the Monkhorst-Pack mesh $4 \times 4 \times 1$ was applied. The plane waves set was restricted by the kinetic energy cutoff 15 Ha. We used the HartwigsenGoedecker-Hutter norm-conserving pseudopotentials [16] in our calculations and the spin-orbit interaction was not taken into account.

The symmetry of wave functions of the obtained valence band states is as follows:

$$
\begin{aligned}
& 10\left(\Gamma_{1}, \Gamma_{3}\right)+10 \Gamma_{5}-10\left(X_{1}, X_{3}\right)+5\left(X_{1}, X_{4}\right)+5\left(X_{2}, X_{3}\right) \\
& \quad-5\left(M_{1}, M_{2}, M_{3}, M_{4}\right)+10 M_{5} .
\end{aligned}
$$

The above equation can be represented also in the following way:

$$
10 \tau_{1}+5 \tau_{3}+5 \tau_{4}
$$


where $\tau_{i}$ denotes a combination of states at high-symmetry points, describing the elementary energy bands given by Eq. (5). It can be seen that Eq. (6) differs from Eq. (5) only by numerical coefficients near the corresponding representation sets. It means that a rearrangement of states occurred under the influence of the crystalline potential at all considered high-symmetry points. Hence, we demonstrated that based upon the empty-lattice approximation one can predict the topology and symmetry of the valence band of artificial periodic system, claiming that it is composed of the elementary energy bands. Note also that there is an equal number of coefficients near the corresponding representation sets in Eq. (6). This suggests that in order to represent Eq. (6) by means of the elementary energy bands, one can use also other elementary energy bands resulting from inducing procedure carried out for more general Wyckoff positions $j(0, y, z)$ and $k(x, a / 2, z)$ in the following way:

$$
\begin{aligned}
& \underbrace{5\left(\Gamma_{1} \oplus \Gamma_{3} \oplus \Gamma_{5}\right)-5\left(2 X_{1} \oplus X_{3} \oplus X_{4}\right)-5\left(M_{1} \oplus M_{3} \oplus M_{5}\right)}_{j(0, y, z)} \\
& +\underbrace{5\left(\Gamma_{1} \oplus \Gamma_{3} \oplus \Gamma_{5}\right)-5\left(X_{1} \oplus X_{2} \oplus 2 X_{3}\right)-5\left(M_{2} \oplus M_{4} \oplus M_{5}\right)}_{k\left(x, \frac{a}{2}, z\right)} .
\end{aligned}
$$

One can see that the coordinates of the positions $j(0, y, z)$ and $k(x, a / 2, z)$ comprise the coordinates of $g_{1}(0,1 / 2, z)$ and $g_{2}(1 / 2,0, z)$, therefore they are an analogue of a passage from the position $c(1 / 8,1 / 8,1 / 8)$ to $e(x, x, x)$ one, connected with the symmetry decrease from $O_{h}^{7}$ to $T_{d}^{2}$, describing the crystals which create the $(\mathrm{GaAs})_{5} /(\mathrm{AlAs})_{5}$ superlattice. Positions $j$ and $k$ describe a plane where the initial bondings between $\mathrm{Ga}$ and $\mathrm{As}$ as well as $\mathrm{Al}$ and $\mathrm{As}$ atoms are located. Hence, we conclude that the numerical coefficients in Eq. (6) play a role of a code where the information about primal host bonding of crystals creating the superlattice is included. Note also that there is an essential difference between the evolution of the actual Wyckoff positions for $\mathrm{A}^{\mathrm{III}} \mathrm{B}^{\mathrm{V}}$ crystals, i.e. $e(x, x, x)$ (from $c(1 / 8,1 / 8,1 / 8)$ ) and for the superlattices based upon those crystals, i.e. $j(0, y, z)$ and $k(x, 1 / 2, z)$ (from $g(0,1 / 2, z)$ ). The difference is due to the fact that there are ten times more atoms in the unit cell of the considered superlattice compared to the unit cell of the host crystals. Therefore there is enough number of states to be described by the higher dimensional elementary energy bands than those of the Wyckoff position $g$. Figure 2 presents the spatial valence electron density distribution in the unit cell of $(\mathrm{GaAs})_{5} /(\mathrm{AlAs})_{5}$ in the planes which are at the same time the position $j_{1}(0, y, z)$ and $k_{1}(x, 1 / 2, z)$, respectively. In these planes there are the lines of the $g_{1}$ and $g_{2}$ positions located, along which arsenic anions attracting gallium electrons are situated. One can see that the maxima of the valence electron density are present in the predicted Wyckoff positions $j$ and $k$.

The band structure calculation results show also that the smallest forbidden energy gap is located in the $\Gamma-Z$ direction and equals $\sim 1 \mathrm{eV}$ which is 


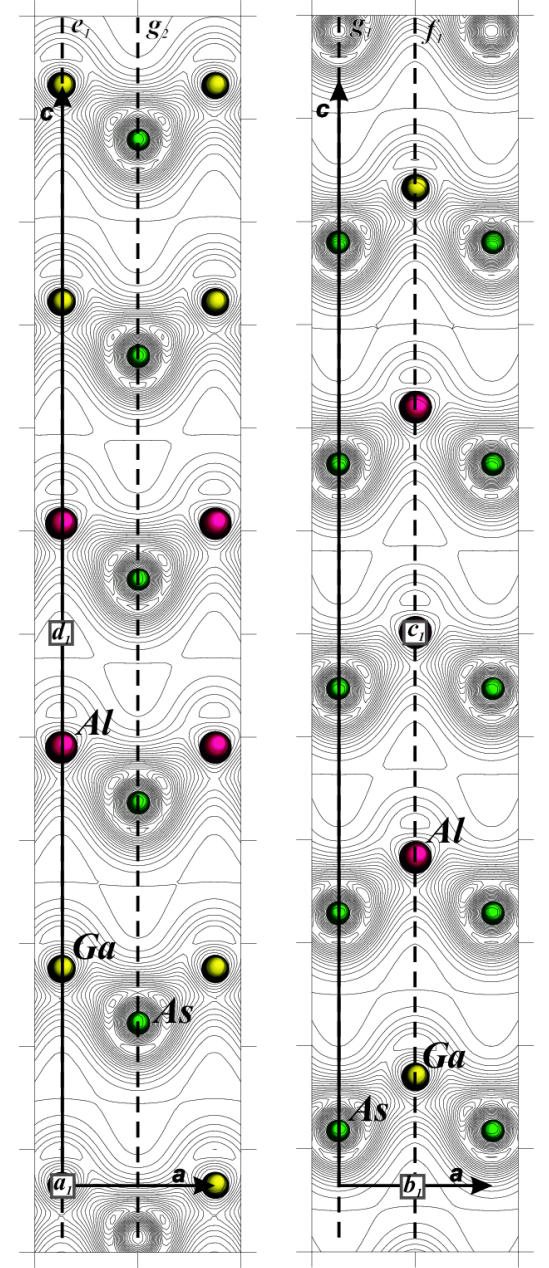

Fig. 2. Cross-sections of the unit cell of $(\mathrm{GaAs})_{5} /(\mathrm{AlAs})_{5}$ with the planes left: $y=0$, which is at the same time the Wyckoff position $j_{1}(x, 0, z)$, right: $y=0.5$ which is at the same time the Wyckoff position $k_{1}(x, 1 / 2, z)$. The Wyckoff positions $a_{1}, b_{1}, c_{1}, d_{1}, e_{1}, f_{1}, g_{1}, g_{2}$ are also displayed.

essentially less than the experimental value $1.9407 \mathrm{eV}$ [17]. The valence band of $(\mathrm{GaAs})_{5} /(\mathrm{AlAs})_{5}$ is composed of 4 groups of minibands. Since the $M(2 \pi / a, 0,0)$ point in the $\mathrm{BZ}$ of the superlattice is the counterpart of $X(2 \pi / a, 0,0)$ in the $\mathrm{BZ}$ of $\mathrm{A}^{\mathrm{III}} \mathrm{B}^{\mathrm{V}}$ crystal creating the superlattice, the gap existing between $X_{1}$ and $X_{3}$ states in the valence band of GaAs (AlAs) crystal is observed as a double gap between the bottom lowest group of branches in the $M$ point and the two twin groups of branches which map for this superlattice the $X_{3}$ state of GaAs crystal (lower gap) and of AlAs crystal (upper gap). Let us remark that in the valence 
band of $(\mathrm{GaAs})_{5} /(\mathrm{AlAs})_{5}$ composed of two kinds of the elementary energy bands having three branches in the $\Gamma$ point (Eq. (8)), we do not observe such distinct elementary energy bands, even for low energy values, as opposed to the host crystals. The lack of such isolation follows directly from the folding procedure of the spectrum in going from the crystal to the superlattice.

\section{Evolution of the elementary energy bands at the creation of the solid solution}

We choose for the discussion the isovalent $\mathrm{A}^{\mathrm{IV}} \mathrm{B}^{\mathrm{VI}}$ crystals which are described by different space symmetry groups: $\operatorname{PbS}\left(O_{h}^{5}\right), \operatorname{SnS}\left(D_{2 h}^{16}\right)$ and the ordered solid solution $\mathrm{Pb}_{0.5} \mathrm{Sn}_{0.5} \mathrm{~S}\left(C_{2 v}^{2}, P 2_{1} m a\right)$ obtained based upon them. $\mathrm{Pb}$ and $\mathrm{Sn}$ atoms are situated uniformly in the structure of this solid solution (see Fig. 3).
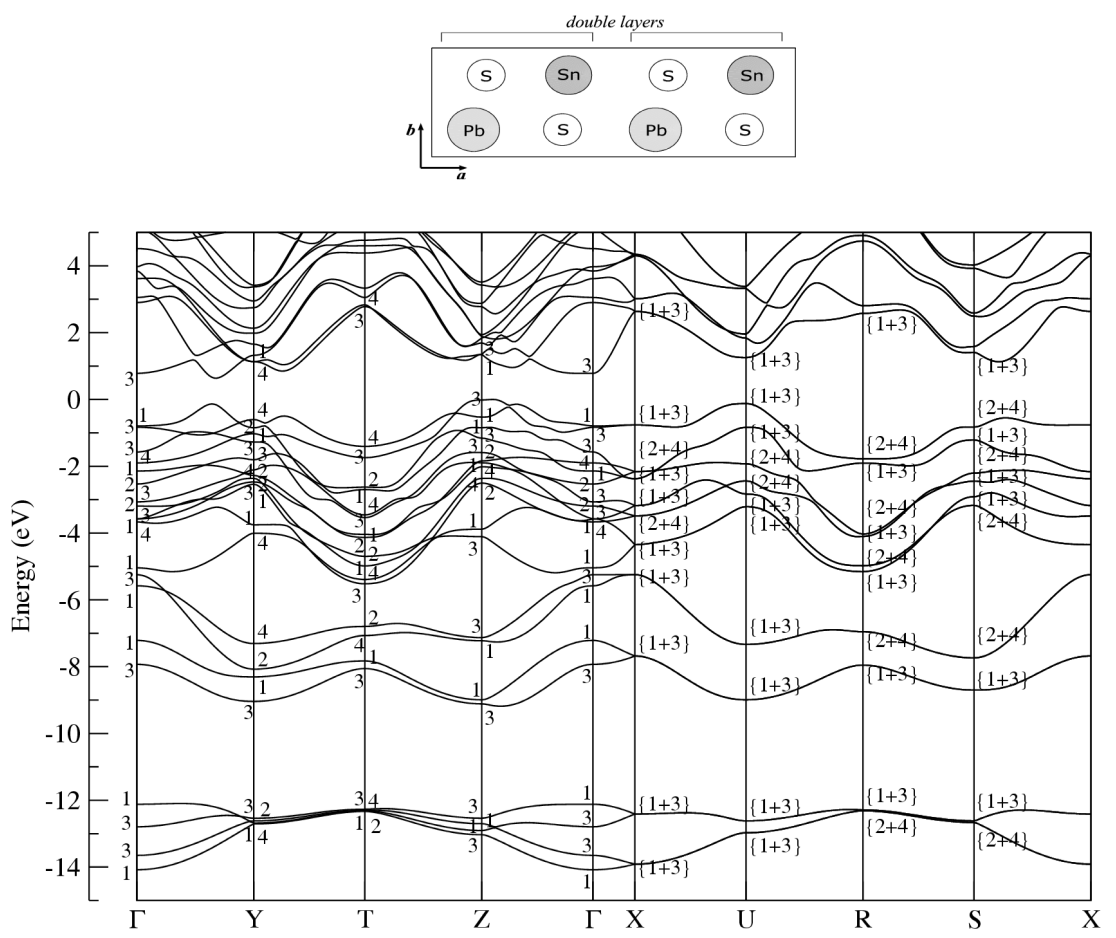

Fig. 3. Top: projection of $\mathrm{Pb}_{0.5} \mathrm{Sn}_{0.5} \mathrm{~S}$ on the $x y$ plane, bottom: its band structure. Calculation details: code - ABINIT [14], pseudopotentials - Hartwigsen-GoedeckerHutter [16], $E_{\text {cut }}=20 \mathrm{Ha}$, integration in the BZ - Monkhorst-Pack $4 \times 4 \times 4$ mesh.

In order to utilize the irreducible and induced representations of the $P m c 2_{1}$ space group presented in Ref. [9] for the symmetry group of this solid solution, one should transform the system of coordinates in the following way: $(X Y Z)\left(P 2_{1} m a\right)=(Z X Y)\left(P m c 2_{1}\right)+(0,1 / 4 /, 1 / 4)$ and, as a consequence, irreducible representations and the coordinates of Wyckoff positions. In the coordinate 
system of $P 2_{1} m a$ the Wyckoff positions for the $C_{2 v}^{2}$ space group are the following:

$$
\begin{aligned}
& a_{1}(x, 1 / 4, z), a_{2}(x+1 / 2,1 / 4,-z+1 / 2), \\
& b_{1}(x, 3 / 4, z), b_{2}(x+1 / 2,3 / 4,-z+1 / 2) .
\end{aligned}
$$

The empty-lattice approximation results enable one to write the closed valence band of $\mathrm{Pb}_{0.5} \mathrm{Sn}_{0.5} \mathrm{~S}$ (after a rearrangement of states in the $\Gamma$ point) as the following set of states:

$$
8\left(\Gamma_{1}, \Gamma_{3}\right)+2\left(\Gamma_{2}, \Gamma_{4}\right)-5\left(T_{1}, T_{3}\right)+5\left(T_{2}, T_{4}\right)-5\left\{S_{1}+S_{2}\right\}+5\left\{S_{2}+S_{3}\right\} .
$$

States in parenthesis are joined due to the time-reversal symmetry. From the irreducible representations of the site-symmetry groups of the Wyckoff positions $a$ and $b$ given by Eq. (9) one can induce the following elementary energy bands which enter the valence band of $\mathrm{Pb}_{0.5} \mathrm{Sn}_{0.5} \mathrm{~S}$ with some numerical coefficients:

$$
\begin{aligned}
\text { from the position } a: & 4\left(\Gamma_{1} \oplus \Gamma_{3}\right)+4\left(T_{1} \oplus T_{3}\right)+4\left\{S_{1}+S_{3}\right\}, \\
& 1\left(\Gamma_{2} \oplus \Gamma_{4}\right)+1\left(T_{2} \oplus T_{4}\right)+1\left\{S_{2}+S_{4}\right\}, \\
\text { from the position } b: \quad & 4\left(\Gamma_{1} \oplus \Gamma_{3}\right)+4\left(T_{2} \oplus T_{4}\right)+4\left\{S_{2}+S_{4}\right\}, \\
& 1\left(\Gamma_{2} \oplus \Gamma_{4}\right)+1\left(T_{1} \oplus T_{3}\right)+1\left\{S_{1}+S_{4}\right\} .
\end{aligned}
$$

Hence, both Wyckoff positions $a$ and $b$ are responsible for the symmetry and topology of this solid solution. There are $\mathrm{Pb}$ and $\mathrm{S}$ atoms situated in the position $a$, while Sn and S atoms are present in $b$. Since all atoms are located in the Wyckoff positions, therefore valence electrons belonging to all of these atoms are responsible for the chemical bonding in this solid solution. The solid solution $\mathrm{Pb}_{0.5} \mathrm{Sn}_{0.5} \mathrm{~S}$ is created based upon $\mathrm{SnS}$ which is described by the space symmetry group $D_{2 h}^{16}$, where the actual is only one Wyckoff position $c(x, 1 / 4, z)^{5}$, therefore a splitting of this position into two actual positions $a$ and $b$ occurs at the symmetry decrease from $D_{2 h}^{16}$ to $C_{2 v}^{2}$. As a consequence, the elementary energy bands consisting of 4 branches in the case of $D_{2 h}^{16}$ group split into two-branch elementary energy bands with the symmetry and topology given by Eq. (11) and observed in the ab initio band structure of the solid solution $\mathrm{Pb}_{0.5} \mathrm{Sn}_{0.5} \mathrm{~S}$ presented in Fig. 3. The symmetry of the obtained elementary energy bands as well as their number in the valence band coincide with that of Eq. (11).

\section{Conclusions}

Based upon the results of empty-lattice approximation for $\mathrm{A}^{\mathrm{IV}}, \mathrm{A}^{\mathrm{III}} \mathrm{B}^{\mathrm{V}}$, and $\mathrm{A}^{\mathrm{IV}} \mathrm{B}^{\mathrm{VI}}$ crystals, we demonstrated that the valence bands of these crystals are composed of the elementary energy bands. To those elementary energy bands correspond the actual Wyckoff positions which can be a point, line or plane. In the case of $A^{\mathrm{IV}} \mathrm{B}^{\mathrm{VI}}$ crystals, the elementary energy bands originate from two actual Wyckoff positions. Such actual positions have a physical meaning, since there is a maximum of the valence electron density located there. We demonstrated an universality of the empty-lattice approximation for the prediction of the topology and symmetry of the valence band of superlattices and ordered solid solutions which 
originate from the discussed crystals at the symmetry decrease of the system. Numerical band structure results as well as the valence electron density distribution confirm the results obtained in the empty-lattice approximation. We showed that the elementary energy bands for $\mathrm{A}^{\mathrm{III}} \mathrm{B}^{\mathrm{V}}$ crystals should be regarded as physically related ones and hence we found an itinerant Wyckoff position along a line joining $\mathrm{A}^{\mathrm{III}}$ and $\mathrm{B}^{\mathrm{V}}$ atoms. This result was used to find the actual Wyckoff positions for superlattices built upon those crystals.

\section{References}

[1] J. Zak, Phys. Rev. Lett. 45, 1025 (1980).

[2] J. Zak, Phys. Rev. B. 25, 1344 (1982).

[3] J. Zak, Phys. Rev. B. 26, 3010 (1982).

[4] L. Michel, J. Zak, Phys. Rev. B. 59, 5998 (1999).

[5] D.M. Bercha, K.Z. Rushchanskii, M. Sznajder, A. Matkovskii, P. Potera, Phys. Rev. B 66, 195203 (2002).

[6] D.M. Bercha, I.V. Slipukhina, M. Sznajder, K.Z. Rushchanskii, Phys. Rev. B 70, 235206 (2004).

[7] F. Bassani, G. Pastori Paravicini, in: Electronic States and Optical Transitions in Solids, Pergamon Press, Oxford 1975.

[8] International Tables for Crystallography, Space Groups Symmetry, Vol. A, D. Reidel Publishing Company, Dordrecht 1983.

[9] O.V. Kovalev, in: Representations of the Crystallographic Space Groups. Irreducible Representations, Induced Representations and Corepresentations, Eds. H.T. Stokes, D.M. Hatch, Gordon and Breach, Amsterdam 1993, p. 390.

[10] A. Garcia, M.L. Cohen, Phys. Rev. B. 47, 4215 (1993).

[11] P. Hohenberg, W. Kohn, Phys. Rev. B 136, 864 (1964).

[12] W. Kohn, L.J. Sham, Phys. Rev. B 140, 1133 (1965).

[13] J.P. Perdew, Y. Wang, Phys. Rev. B 45, 13244 (1992).

[14] X. Gonze, J.-M. Beuken, R. Caracas, F. Detraux, M. Fuchs, G.-M. Rignanese, L. Sindic, M. Verstraete, G. Zerah, F. Jollet, M. Torrent, A. Roy, M. Mikami, Ph. Ghosez, J.-Y. Raty, D.C. Allan, Comput. Mater. Science 25, 478 (2002); The ABINIT code is a common project of the Universite Catholique de Lovain, Corning Incorporated, and other contributors (URL http://www.abinit.org).

[15] H.J. Monkhorst, J.D. Pack, Phys. Rev. B 13, 5188 (1976).

[16] C. Hartwigsen, S. Goedecker, J. Hutter, Phys. Rev. B 58, 3641 (1998).

[17] V.G. Litovchenko, D.V. Korbutyak, S. Krylyuk, H.T. Grahn, K.H. Ploog, Phys. Rev. B 55, 10621 (1997). 